\title{
Long-time tails do not necessarily imply self-organized criticality or the breakdown of the standard transport paradigm
}

\author{
John A. Krommes \\ Princeton University, P.O. Box 451, Princeton, New Jersey 08543-0451, USA \\ Maurizio Ottaviani \\ Département de Recherches sur la Fusion Contrôlée, Commissariat à l'Energie Atomique, \\ Centre de Cadarache, 13108 St. Paul lez Durance, France
}

(May 14, 1999)

\begin{abstract}
Numerical measurements and analytical studies are performed on a stochastic model with features relevant to plasma confinement. Although the model lacks crucial features of self-organized criticality (SOC) and its transport can be computed by standard techniques, it nevertheless exhibits intermittency and algebraic time correlations. This suggests that SOC need not be the explanation for observed long-time tails in experimental fluctuation data. Arguments based on the renormalized spectral balance equation, and simulation of a standard nonlinear paradigm, predict a range of Hurst exponents in reasonable agreement with the observations without invoking submarginal dynamics.
\end{abstract}

PACS: 52.35.Ra, 05.65+b

Recently Carreras and coworkers have made interesting measurements of long-time correlations in various experimental plasma fluctuation data. ${ }^{1,2}$ The results suggest that certain two-time correlation functions possess algebraic tails of the form $|\tau|^{-\beta}(0<\beta<1)$ for time lags $\tau$ long compared to a characteristic correlation time of the turbulence. It has been remarked ${ }^{2,3}$ that such behavior is compatible with the paradigm ${ }^{4}$ of self-organized criticality (SOC), ${ }^{5}$ and asserted ${ }^{6}$ that such tails imply the breakdown of the standard transport model. However, in the present paper we argue that long-time tails need neither be related to SOC nor imply that standard transport calculations must necessarily be forsaken, and we predict nontrivial $\beta$ 's without invoking SOC arguments.

The precise definition of the "SOC paradigm" seems to vary somewhat with author. In the original papers, ${ }^{7,5}$ which used discrete "sandpile" models as illustrations, SOC was defined to be a scale-invariant final state achieved without the necessity of fine-tuning a parameter (in contrast to the situation in critical phenomena ${ }^{8}$ ). Perturbations to that state (by very slow forcing) resulted in avalanches (transport events) of all scales. In the papers that have suggested possible relevance to plasma transport, ${ }^{9,10,4}$ particular emphasis has been placed on the need for nontrivial linear thresholds, the importance of submarginal dynamics, and the intermittent nature of the fluctuations. Note that it is vacuous to define an SOC system merely as one that exhibits scale similarity, since that embraces a uselessly large class of systems with vastly different properties. ${ }^{11}$

We first discuss numerical solutions of a particular stochastic model, defined by Eq. (1) below, that generates measurable turbulent fluctuations for a conserved scalar field that we shall call "temperature" $\widetilde{T}$ (it could equally well be called "density"), a velocity field $\widetilde{u}$, and the associated flux $\widetilde{\Gamma}$ of $\widetilde{T}$ through the system. (The tilde denotes a random variable. Quantities sans tildes denote the statistical average e.g., $T \equiv\langle\widetilde{T}\rangle$.) Representative data are presented in Figs. 1 and 2. Fig. 1 shows the measured probability density function (PDF) of the turbulent flux $\widetilde{\Gamma}_{\mathrm{tb}}(x, t) \doteq(\delta u \delta T)(x, t)$, where, e.g., $\delta T \doteq \widetilde{T}-T$. The flux is clearly intermittent, with a higher than Gaussian probability of large-amplitude events. Fig. 2a shows that the two-time correlation of $\widetilde{T}$ exhibits an almost perfect long-time tail with $\beta \approx \frac{1}{2}$ over more than 30 correlation times of $\widetilde{u}$. This is corroborated by analysis of the Hurst exponent ${ }^{12,2} H=1-\frac{1}{2} \beta$; as shown in Fig. 2 b, we measure $H \approx 0.79$. Such intermittent, long-time behavior is in some respects qualitatively similar to the recent experimental measurements. ${ }^{2}$ However, although the data may appear to be consistent with an SOC paradigm, we shall show that the model does not contain crucial features of SOC. Therefore, as we will discuss, our results support the suggestion of Carreras et al. ${ }^{3}$ that an interpretation of the observations alternative to SOC may exist.

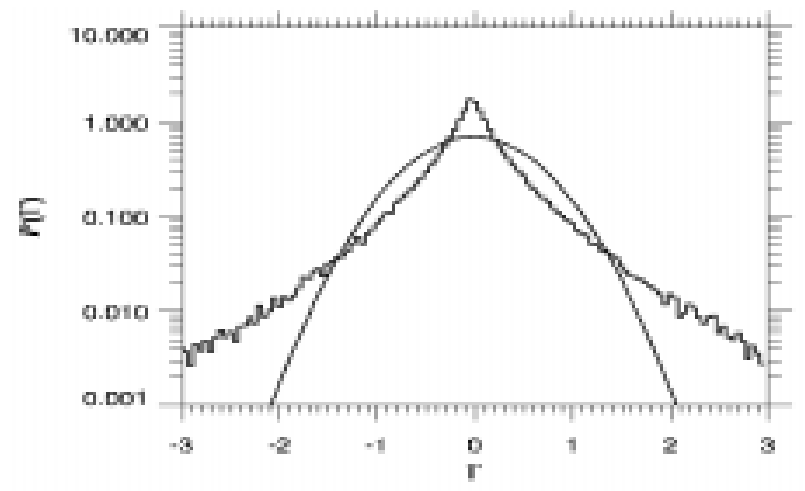

FIG. 1. The PDF of the turbulent flux $\widetilde{\Gamma}_{\mathrm{tb}}$. Comparison with a reference Gaussian with the same mean and variance confirms the presence of intermittency. [ $\widetilde{T}$ (PDF not shown) is also highly intermittent; the analysis of Ref. 13 is inapplicable.] 

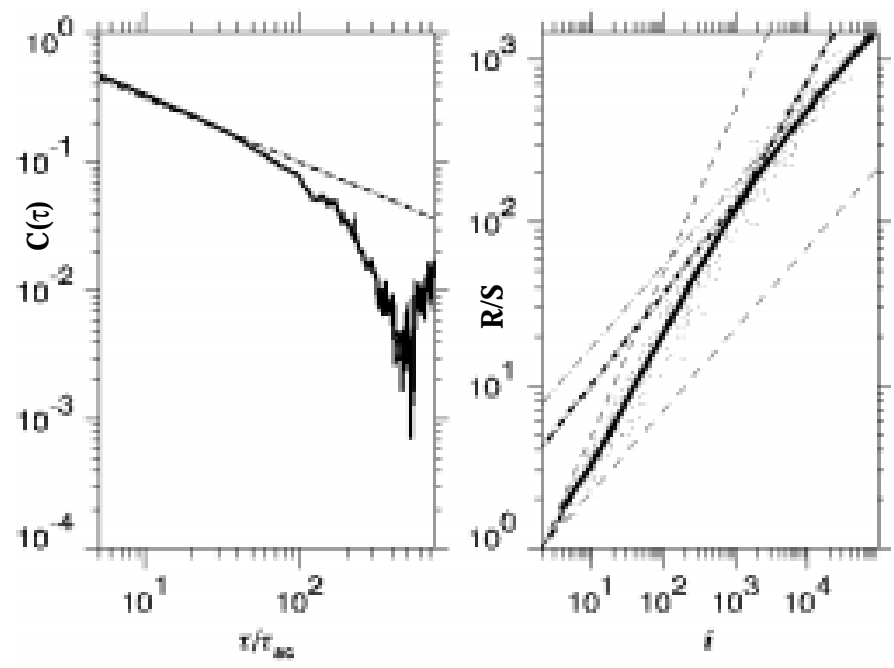

FIG. 2. (a) Correlation function of the temperature evolved according to Eq. (1) and measured at $x=\frac{1}{4}$. The time lag $\tau$ is in units of the measured autocorrelation time $\tau_{\mathrm{ac}}$ [cf. Eq. (2)] of the velocity field. The dashed line has slope $-\frac{1}{2}$. Note the transition to exponential decay at $\tau / \tau_{\text {ac }} \lesssim 100$. (b) Analysis of the Hurst exponent $H$ for the temperature field by the $R / S$ method (cf. Refs. 12 and 2). Each integer time lag $i$ corresponds to $5 \tau_{\text {ac }}$ 's. Data are plotted every quarter octave, with 10 (random) samples per lag. The chain-dotted straight-line fits correspond to the slopes $H \approx 0.79\left(\tau<\tau_{E}\right)$ and $H \approx 0.59\left(\tau \gg \tau_{E}\right)$. The solid line is the best fit of a ratio of fifth-order polynomials, intended to qualitatively emphasize the existence of several regimes. Two reference lines of slope $H=\frac{1}{2}$ and one of slope $H=1$ are also plotted.

The fluctuation data just described stem from a simple model of random passive advection. The model comprises the 1D boundary-value problem

$$
\partial_{t} \widetilde{T}(x, t)+\partial_{x} \widetilde{\Gamma}(x, t)=0 \quad(0<x<1),
$$

where $\widetilde{\Gamma}(x, t) \doteq \widetilde{\Gamma}_{\mathrm{tb}}+\widetilde{\Gamma}_{\mathrm{cl}}$ is the total thermal flux, composed of the turbulent flux $\widetilde{\Gamma}_{\mathrm{tb}}$ and the classical flux $\widetilde{\Gamma}_{\mathrm{cl}} \doteq$ $-\mathcal{R}^{-1} \partial_{x} \widetilde{T}$, where $\mathcal{R}$ is a constant. The random velocity $\widetilde{u}$ is taken to be a centered Gaussian stationary in time and homogeneous in space, specified by taking the covariance $U(\rho, \tau) \doteq\langle\delta u(x+\rho, t+\tau) \delta u(x, t)\rangle$ to be

$$
U(\rho, \tau)=\bar{u}^{2} \exp \left(-|\tau| / \tau_{\mathrm{ac}}\right) \exp \left(-\frac{1}{2} \rho^{2} / L_{\mathrm{ac}}^{2}\right)
$$

with $\bar{u}=1, \tau_{\mathrm{ac}}$ and $L_{\mathrm{ac}}$ being adjustable parameters. We always impose $T(1)=0$, but can study either the "fixed" boundary condition (b.c.) $\widetilde{T}(0)=1$ [note that Eq. (1) is linear in $\widetilde{T}]$ or the flux b.c. $\widetilde{\Gamma}(0, t)=\widetilde{\Gamma}_{0}(t)$, where $\widetilde{\Gamma}_{0}(t)$ is a specified function that may contain both a mean and a fluctuating part. This model shares with realistic confinement experiments the properties: (i) the mean profile $T(x, t)$ (independent of $t$ in a steady state) is not specified, but is free to evolve; (ii) the correlation scales of the velocity field can be taken to be small ("microscopic") with respect to macroscopic values by choosing $\tau_{\mathrm{ac}}$ and $L_{\mathrm{ac}}$ to be much smaller than 1; (iii) the mean temperature obeys $\partial_{t} T+\partial_{x} \Gamma=0$, which in a steady state reduces to the statement that the total (mean) flux is a conserved constant, independent of $x$; (iv) the fluctuations are not forced by arbitrary additive noise on the right-hand side of Eq. (1), but by multiplicative noise ${ }^{14}$ of the usual advective variety.

We choose a 1D model in order to expedite numerical solution and accumulation of adequate statistics. The random nature of $\widetilde{u}$ is intended to introduce effects that would in reality arise from multidimensional nonlinear interactions. Unfortunately, the desire to maintain the conservation law, ensured by writing $\partial_{x}(\widetilde{u} \widetilde{T})$ rather than $\widetilde{u} \partial_{x} \widetilde{T}$ in Eq. (1), requires that the dynamics be compressible $\left(\partial_{x} \widetilde{u} \neq 0\right)$ for $L_{\mathrm{ac}} \neq \infty$. That is an important difference from more realistic models whose nonlinearities are built from the incompressible (for constant $B$ ) $\boldsymbol{E} \times \boldsymbol{B}$ velocity. Nevertheless, useful qualitative insights can be obtained.

Although Eq. (1) is dynamically linear, it is stochastically nonlinear ${ }^{15}$ (involving the product of the random variables $\widetilde{u}$ and $\widetilde{T}$ ). Dynamically nonlinear saturation processes that would occur in self-consistent equations are replaced here by the imposition of a constant $\bar{u}$. $\widetilde{T}$ evolves to a statistically steady state arising from the balance between the random advection and the classical dissipation.

We noted in paragraph 2 that standard models of SOC require nontrivial linear thresholds and submarginal dynamics, and that those features have been associated with intermittent transport and long-time tails. However, with the velocity specified by Eq. (2), the present model possesses no threshold; fluctuations are always present. It is possible to generalize the model to include the effects of either supermarginal or submarginal dynamics, as described in Ref. 14. That is specifically not done here in order to emphasize that intermittency and long-time tails can result even in the absence of more interesting, especially submarginal, fluctuation dynamics.

The representative data described here correspond to the parameters $\mathcal{R}=50, \tau_{\mathrm{ac}}=0.02, L_{\mathrm{ac}}=0.04$, and to fixed b.c.'s. (Similar results are obtained for flux b.c.'s.) One can predict the observed exponent $\beta \approx \frac{1}{2}$ by the following argument. We begin with the exact equation for $\delta T$ :

$$
\partial_{t} \delta T+\partial_{x}(\delta u \delta T)-\mathcal{R}^{-1} \delta T^{\prime \prime}=-\partial_{x}(\delta u T)+\partial_{x} \Gamma_{\mathrm{tb}} .
$$

This can be formally solved in terms of the random infinitesimal response function $\widetilde{R}\left(x, t ; x^{\prime}, t^{\prime}\right)$, which is Green's function for the left-hand side of Eq. (3). Thus $\delta T(x, t)=$ $-\widetilde{R} \star \partial_{x}\left(\delta u T-\Gamma_{\mathrm{tb}}\right)$, where $\star$ denotes convolution in space and time and an arbitrary initial condition has been ignored, as we wish to discuss the long-time steady state. If the statistics were strictly homogeneous, the term $\partial_{x} \Gamma_{\mathrm{tb}}$ would rigorously vanish. Although we generate a homogeneous velocity field, $\delta T$ is not exactly homogeneous because of the b.c.'s. However, for the current parameters the deviation from homogeneity occurs only in thin boundary layers near the walls, which we shall ignore. We now form the correlation function $C(\tau) \doteq\langle(\delta T / \bar{T})(\tau)(\delta T / \bar{T})(0)\rangle$, where $\bar{T}$ is a suitable reference value characteristic of the interior:

$$
C(\tau) \approx \int_{-\infty}^{t} d \bar{t} \int_{-\infty}^{t^{\prime}} d \bar{t}^{\prime} \int_{0}^{1} d \bar{x} \int_{0}^{1} d \bar{x}^{\prime} R(x, t ; \bar{x}, \bar{t})
$$




$$
\times R\left(x, t^{\prime} ; \bar{x}^{\prime}, \bar{t}^{\prime}\right) \partial_{\bar{x}} \partial_{\bar{x}^{\prime}} U\left(\bar{x}, \bar{t} ; \bar{x}^{\prime}, \bar{t}^{\prime}\right)[T(\bar{x}) / \bar{T}]\left[T\left(\bar{x}^{\prime}\right) / \bar{T}\right],
$$

where we approximated $\langle\widetilde{R} \widetilde{R} \delta u \delta u\rangle \approx\langle\widetilde{R}\rangle\langle\widetilde{R}\rangle\langle\delta u \delta u\rangle=$ $R R U(R \doteq\langle\widetilde{R}\rangle)$. We consider long times, $t>$ $\min \left(\tau_{\mathrm{ac}}, L_{\mathrm{ac}} / \bar{u}\right)$, so $U(\rho, \tau) \approx \bar{u}^{2} \delta\left(\tau / \tau_{\mathrm{ac}}\right) \delta\left(\rho / L_{\mathrm{ac}}\right)$. Upon introducing Fourier transforms in space and time, one finds

$$
C(\tau) \approx \tau_{\mathrm{ac}} L_{\mathrm{ac}} \bar{u}^{2} \int_{-\infty}^{\infty} \frac{d k}{2 \pi} \int_{-\infty}^{\infty} \frac{d \omega}{2 \pi} e^{-i \omega \tau} k^{2}\left|R_{k \omega}\right|^{2} .
$$

We will find that the wave-number integral is dominated by the small $k$ 's $\left(k L_{\mathrm{ac}} \ll 1\right)$. For those $k$ 's, it is adequate to make the usual diffusive approximation $R_{k \omega} \approx$ $\left[-i\left(\omega+i k^{2} D\right)\right]^{-1}$, where $D=D_{\mathrm{tb}}+D_{\mathrm{cl}}$ is the sum of the turbulent diffusion coefficient $D_{\mathrm{tb}}$ and the classical coefficient $D_{\mathrm{cl}}=\mathcal{R}^{-1}$. The frequency integral can then be performed by residues; one finds

$$
C(\tau)=\frac{1}{2 \sqrt{4 \pi}}\left(\frac{D_{\mathrm{q}}}{D}\right)^{3 / 2}\left(\frac{L_{\mathrm{ac}}}{\bar{u} \tau_{\mathrm{ac}}}\right)\left(\frac{\tau_{\mathrm{ac}}}{|\tau|}\right)^{1 / 2},
$$

where $D_{\mathrm{q}}$ is the quasilinear value ${ }^{16} D_{\mathrm{q}} \doteq \bar{u}^{2} \tau_{\text {ac }}$.

One expects that finite-size effects should contaminate the power-law tail on the energy confinement time scale $\tau_{E}=a^{2} / D$, where $a^{-1}=\pi$ (the fundamental wave number for a sine series satisfying the b.c.'s). Direct measurement of $\tau_{E}$ leads to $\tau_{E} / \tau_{\mathrm{ac}} \approx 200$. That is in reasonable agreement with the observed transition from power-law to exponential decay, as shown in Fig. 2a.

One pragmatic difficulty with the measurement of $H$ has to do with finite-size effects. For very long lags, $H$ should converge to 0.5 (exponential decay). A tendency in that direction is confirmed by fitting a ratio of fifth-degree polynomials through the $\log -\log$ data, showing noticeable curvature. Attempts to fit straight lines through the early $\left(\tau \lesssim \tau_{E}\right)$ and late $\left(\tau \gg \tau_{E}\right)$ portions of the data lead to substantially different $H$ 's: $H \approx 0.79$ for the early times, $H \approx 0.59$ for the late times (the time series is too short to achieve asymptotic scaling). In general, measurement of $H$ is delicate when very large time lags are involved.

Even though $\widetilde{T}$ possesses long-time correlations, the thermal flux can be estimated by standard means; one can show that even a quasilinear calculation predicts reasonable results. For this problem, the "standard transport paradigm" does not need to be abandoned. To understand this, it is important to distinguish Green-Kubo integrands (whose $\tau$ integrals are the transport coefficients) from correlation functions of the dependent variables such as $\widetilde{T}$. The former can decay on a microscopic time scale, as they can be dominated by wave numbers in the energy-containing range (which define the typical $\tau_{\mathrm{ac}}$ ).

We have thus shown that at least one stochastic model with small $L_{\mathrm{ac}}$ but lacking crucial features of SOC predicts a long-time algebraic tail. This raises the possibility that the long-time tails in experimental data may have nothing to do with SOC; note that the effective dynamical model underlying the experimental measurements is unknown. However, one may justly wonder to what extent the results generalize to real multidimensional models with self-consistent nonlinear dynamics. This is a very difficult question to which we have no definitive answer. However, some preliminary general remarks can be made.

We now imagine that the plasma obeys a generic, quadratically nonlinear, self-consistent equation for some quantity $\psi$. (Some support for this hypothesis is given by mode-coupling analysis ${ }^{17}$ of numerical gyrokinetic fluctuation data.) Rigorous statistical renormalization ${ }^{18}$ leads to the balance equation ${ }^{19} C_{\boldsymbol{k}, \omega}=\left|R_{\boldsymbol{k}, \omega}\right|^{2} F_{\boldsymbol{k}, \omega}$, where $C$ is the two-point correlation function of $\psi, F$ is the covariance of a well-defined internally produced nonlinear noise, $R$ is again the mean infinitesimal response function, and stationary, homogeneous, isotropic statistics have been assumed; note the similarity to Eq. (4). Of course, this balance is intricately self-consistent because $F$ depends at least on $C$, and $R$ is self-consistently constructed from both $C$ and $R$. [For explicit formulas in the direct-interaction approximation (DIA), cf. Ref. 19.] Nevertheless, one has

$$
C(\tau)=\int \frac{d^{d} \boldsymbol{k}}{(2 \pi)^{d}} \int_{-\infty}^{\infty} \frac{d \omega}{2 \pi} e^{-i \omega \tau}\left|R_{\boldsymbol{k}, \omega}\right|^{2} F_{\boldsymbol{k}, \omega} .
$$

If one assumes that $F_{\boldsymbol{k}}(\tau)$ is short-ranged, one may approximate $F_{\boldsymbol{k}, \omega} \approx F_{\boldsymbol{k}, 0} \equiv F_{\boldsymbol{k}}$. We postulate a generalized diffusive law, $R_{\boldsymbol{k}, \omega} \approx\left[-i\left(\omega+i k^{2 m} \widehat{D}\right)\right]^{-1}$. Then $C(\tau)=\frac{1}{2}(2 \pi)^{-d} \int d^{d} \boldsymbol{k} e^{-k^{2 m} \widehat{D}|\tau|} F_{\boldsymbol{k}}\left(k^{2 m} \widehat{D}\right)^{-1}$. If one postulates that $F_{\boldsymbol{k}}=\mathcal{O}\left(k^{2 n}\right)$ as $k \rightarrow 0$, then one finds that $C(\tau) \sim|\tau|^{-\beta}$, with $\beta=\left(\frac{1}{2} d+n-m\right) / m$. For $d=1$ and $n=m=1$, this reproduces the results of the small$L_{\text {ac }}$ stochastic model, namely $\beta=\frac{1}{2}, H=\frac{3}{4}$. For $d=2$ (the most reasonable choice for a generic problem of quasiisotropic plasma transport in a strong magnetic field) and $n=m$, one has $\beta=1 / m, H=1-1 / 2 m$.

If one analyzes Hasegawa-Mima (HM) dynamics [a generic nonlinear model of drift waves with $d=2(\psi=$ potential)] with second-order statistical closure, one finds $n=m=2$ if the spectra are assumed to be localized around some $\bar{k} \gg k$, so one again predicts $\beta=\frac{1}{2}$, $H=\frac{3}{4}$. However, the HM paradigm, with adiabatic electrons, is an inadequate representation of the actual physics. A better paradigm for edge fluctuations is the Hasegawa-Wakatani (HW) model. ${ }^{20,21}$ We have simulated the $2 \mathrm{D}$ version of that model (fixed $k_{\|}$) in the absence of magnetic shear ${ }^{22}$ at resolution $512^{2}$ (before dealiasing) and adiabaticity parameter ${ }^{20} \alpha=0.1$ for $t / \tau_{\mathrm{ac}} \gtrsim 1000$, $\tau_{\text {ac }}$ being the measured density correlation time. (That is $\approx 1 / 2$ of the $\tau_{E}$ based on the measured particle flux and $k_{x, \min }=0.01$.) Direct calculation of the two-time density autocorrelation function reveals (Fig. 3a) an initial decay of the form $\exp \left(-\frac{1}{2}\left(\tau / \tau_{*}\right)^{2}\right)$ [corresponding to a microscopic autocorrelation time $\left.\tau_{\mathrm{ac}}^{*} \doteq(\pi / 2)^{1 / 2} \tau_{*}\right]$ and some evidence of a tail, slightly steeper than $\left(\tau / \tau_{*}\right)^{-1}$, that persists for $\tau / \tau_{\mathrm{ac}}^{*} \gtrsim 4-6$ until it is disguised by a weak oscillation, then statistical noise. (The vorticity does not evince such a tail.) A $d=2, \beta=1$ tail corresponds to $n=m=1, H=\frac{1}{2}$ and is consistent with turbulent density diffusion. Note that a 
tail steeper than $\tau^{-1}$, or $\tau^{-1}$ with oscillations, has a finite $\tau_{\text {ac }} \ll \tau_{E}$. An $R / S$ measurement (Fig. 3b) is compatible with $H=\frac{1}{2}$ (which does not distinguish between shorttime decay and a $\tau^{-1}$ tail). (Note that $R / S$ measurements on limited data samples are difficult because of statistical noise, which spuriously enhances the predicted $H$, as do measurements in pre-asymptotic regimes.)
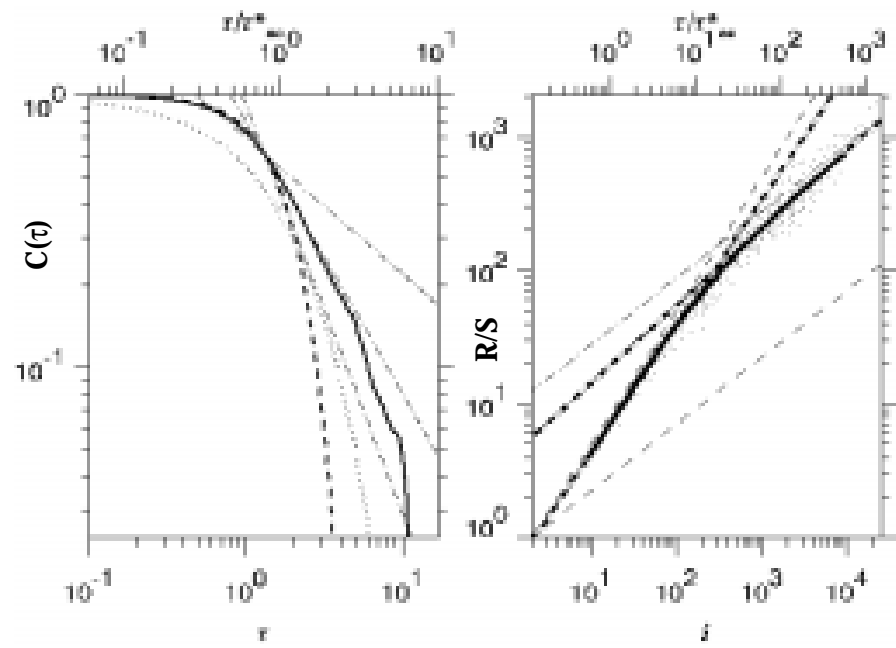

FIG. 3. (a) Time-lagged density autocorrelation function for the 2D HW model. Solid line: simulation. Dashed line: $\exp \left(-\frac{1}{2}\left(\tau / \tau_{*}\right)^{2}\right)$ $(\tau *=1.3)$. Dotted line: $\exp \left(-\tau / \tau_{\mathrm{ac}}^{*}\right)$ for $\tau_{\mathrm{ac}}^{*}=(\pi / 2)^{1 / 2} \tau *$. Chain-dotted lines: $\left(\tau / \tau_{*}\right)^{-\beta}$ for (top to bottom) $\beta=\left(\frac{1}{2}, 1, \frac{3}{2}\right)$. (b) Corresponding $R / S$ data (5 samples per octave). Left-hand slope $(2 \leq i \leq 200): H=0.95$. Right-hand slope $(500 \leq i \leq 10000)$ : $H=0.58$. Solid line: ratio of fifth-order polynomials (right-most derivative $H=0.55$ ). (The identical data with 4 samples per octave predicts $H=0.62$, showing variability due to random sampling of the database for short lags.)

For comparison, the quoted experimental values ${ }^{2}$ for fluctuations within the last closed flux surface lie mostly in the range $0.62 \pm 0.01<H<0.72 \pm 0.07$. Although the agreement of these values with the above estimates $\frac{1}{2} \leq H \leq \frac{3}{4}$ is intriguing, we do not claim to have modelled the observed values; the paradigms are too simple. Also, the analysis of Eq. (7) may fail for various reasons, including lack of isotropy, the possible presence of branch cuts, corrections to asymptotic scaling, and the influence of strongly non-Gaussian statistics. Note that although highly non-Gaussian effects need not be well described by the DIA, ${ }^{23}$ the Realizable Markovian Closure, ${ }^{24}$ a close relative of the DIA and a standard transport paradigm, has been shown to be an excellent approximation for the calculation of the turbulent flux in the HW model. ${ }^{20,21}$ As one consistency check, the calculations leading to the $|\tau|^{-d / 2}$ tail predicted for $n=m=1$ are compatible with a wellknown result for thermal equilibrium many-particle fluids and plasmas; ${ }^{25}$ note that the balance equation is compatible with the fluctuation-dissipation theorem. ${ }^{26}$

In conclusion, both passive stochastic and dynamically self-consistent models that do not possess crucial features of SOC systems (e.g., fluctuation thresholds and submarginal dynamics) can exhibit intermittency and long-time correlations that bear some qualitative resemblance to those observed experimentally but do not necessarily imply the breakdown of the standard transport paradigm. Realistic tails can be produced from long-wavelength fluctuations unrelated to SOC. Further research is required to determine whether or not an SOC paradigm is relevant to the interpretation or calculation of plasma transport.

\section{ACKNOWLEDGMENTS}

One of us (JAK) acknowledges the gracious hospitality of the Department of Controlled Fusion Research, Cadarache, where this work was begun. The research was supported in part by U.S. Department of Energy Contract No. DEAC02-76-CHO-3073.

${ }^{1}$ B. A. Carreras, B. van Milligen, M. A. Pedrosa et al., Phys. Rev. Lett. 80, 4438 (1998).

2 B. A. Carreras, B. P. van Milligen, M. A. Pedrosa et al., Phys. Plasmas 5, 3632 (1998).

3 The authors of Ref. 2 were appropriately cautious in their conclusions. They remarked that their measurements were "consistent with the SOC paradigm of turbulent transport. However, it does not prove that this model offers the only explanation... . At this point, we are not aware of other dynamical mechanisms that may provide an alternative answer, but it may exist."

4 B. A. Carreras, D. Newman, V. E. Lynch, and P. H. Diamond, Plasma Phys. Rep. 22, 740 (1996).

${ }^{5}$ H. J. Jensen, Self-Organized Criticality (Cambridge U. Press, Cambridge, UK, 1998).

${ }^{6}$ B. A. Carreras, Bull. Am. Phys. Soc. 43, 1920 (1998).

7 P. Bak, C. Tang, and K. Wiesenfeld, Phys. Rev. A 38, 364 (1988).

${ }^{8}$ N. Goldenfeld, Lectures on Phase Transitions and the Renormalization Group (Addison-Wesley, Reading, MA, 1992).

9 P. H. Diamond and T.-S. Hahm, Phys. Plasmas 2, 3640 (1995).

10 D. E. Newman, B. A. Carreras, P. H. Diamond, and T.-S. Hahm, Phys. Plasmas 3, 1158 (1996).

11 A. Vespignani and S. Zapperi, Phys. Rev. E 57, 6345 (1988).

12 B. B. Mandelbrot, Multifractals and $1 / f$ Noise (Springer, New York, 1998).

13 B. A. Carreras, C. Hidalgo, E. Sánchez et al., Phys. Plasmas 3, 2664 (1996).

14 J. A. Krommes, Phys. Plasmas 4, 1342 (1997).

${ }^{15}$ R. H. Kraichnan, J. Math. Phys. 2, 124 (1961), erratum: J. Math. Phys. 3, 205 (1962).

16 J. A. Krommes and R. A. Smith, Ann. Phys. 177, 246 (1987).

17 H. E. Mynick and S. E. Parker, Phys. Plasmas 2, 1217 (1995).

18 P. C. Martin, E. D. Siggia, and H. A. Rose, Phys. Rev. A 8, 423 (1973).

19 J. A. Krommes, in Handbook of Plasma Physics, edited by A. A. Galeev and R. N. Sudan (North-Holland, Amsterdam, 1984), Vol. 2, Chap. 5.5, p. 183.

20 G. Hu, J. A. Krommes, and J. C. Bowman, Phys. Plasmas 4, 2116 (1997).

21 J. A. Krommes, Phys. Rep. 283, 5 (1997).

22 Note that although the 3D HW system, including $k_{\|}=0$ modes, can support submarginal turbulence [J. A. Krommes, Plasma Phys. Control. Fusion 41, A641 (1999)], that fact is irrelevant in the absence of shear and in 2D.

23 J. A. Krommes, Phys. Rev. E 53, 4865 (1996).

${ }^{24}$ J. Bowman, J. A. Krommes, and M. Ottaviani, Phys. Fluids B 5, 3558 (1993).

25 J. A. Krommes and C. Oberman, J. Plasma Phys. 16, 229 (1976).

${ }^{26}$ J. A. Krommes, Phys. Fluids B 5, 650 (1993). 